\title{
Translational medicine in China I: Perspectives from Chinese physicians and scientists
}

\author{
JIANG ChengYu \\ Department of Biochemistry and Molecular Biology, Peking Union Medical College, \\ Chinese Academy of Medical Sciences, Beijing 100005, China
}

Received November 20, 2011

Citation: Jiang C Y. Translational medicine in China I: Perspectives from Chinese physicians and scientists. Sci China Life Sci, 2011, 54: 1071-1073, doi: 10.1007/s11427-011-4260-0

Ever since Dr. Elias Zerhouni, then director of the National Institutes of Health, proposed "The NIH Roadmap" in 2003 [1], life sciences and medical fields worldwide have emphasized translational medical research. In this special topic, we have invited distinguished physicians and scientists to offer their perspectives on translational medical research in China.

Minister of health, scientist, and physician Dr. Chen Zhu, invited by our general editor Prof. Zhu ZuoYan, indicates that translational medicine is a people-oriented medical practice (focusing on patients and the general population). While rapid industrialization and urbanization have brought challenges to China's health system, Dr. Chen predicts that the development of translational medicine will accelerate disease control and health solutions with an emphasis on proper principles.

Echoing Dr. Francesco Marincola's “Translational Medicine: A Two-Way Road" [2], Dr. Zhong NanShan, a physician and scientist specializing in respiratory diseases who made significant contributions during the 2003 SARS outbreak, welcomes the impressive development of translational medicine from the traditional "bench to bedside" and "empirical to evidence-based" practice to a practice of "bedside to bench to bedside." He describes three successful cases of translation from traditional Chinese medicine: the use of artemisinin in the management of malaria, the use of arsenic in the management of leukemia, and artificial infection with malaria parasites for lung cancer therapy.

email: Jiang@pumc.edu.cn
A scientist and physician in the field of cardiovascular disease, Dr. Liu DePei reviews classical stories of patient-oriented translational medicine, from the Framingham Heart Study to the development of statins to percutaneous coronary intervention treatment. Prof. Liu, the president of the Chinese Academy of Medical Sciences and Peking Union Medical College, notes two important aspects of translational medicine-translational medical research centers and talents - and summarizes the current situation in China. Prof. Liu proposes that "9P-3P" medicine should guide the development of translational research.

Adenomatous polyposis coli may have been the first gene to be linked with translational medicine by James Geraphty in 1996 [3]. Dr. Zeng YiXin, oncologist and president of the Cancer Hospital Affiliated with Sun Yat-sen University, summarizes recent advances in molecular targeted tumor therapy in China, which may be the practice that has benefited most significantly from translational medical research. Dr. Zeng foresees that "looking for targets more specific to tumor cells" and "individualized therapy under the guidance of molecular markers to improve the effectiveness of treatment" shall be the future trends.

Although translational medicine seems to be a new discipline, the understanding of its effects can be traced to Dr. Liu Shih-Hao, the founder of endocrinology in China, in the early part of the last century. Dr. Li NaiShi, a physician from Peking Union Medical College Hospital, discusses the application of translational medicine in the research, teaching and clinical work of Dr. Liu, who is particularly re- 
nowned for his early work in calcium and phosphorus metabolism. Dr. Liu Shih-Hao's early translational medical research work "Treatment of renal osteodystrophy with dihydrotachysterol (A.T. 10) and iron" published on Science in 1942 was still cited last year by Dr. S. M. Moe in his book Definition and Classification of Renal Osteodystrophy and Chronic Kidney Disease-mineral Bone Disorder [4].

Translational medicine aims to bridge basic science research and clinical practice. In recent years, China has published many important discoveries related to the research of translational medicine. Some of the major themes touched by these publications include: research into mechanisms of diseases with the aim of identifying new therapeutic targets or improved therapeutic strategies [5-18], "omics" approaches to human diseases [19-26], testing of new therapeutic methods in animal models [27-31], research into the mechanisms of host immune defense against infections [32-48], and regenerative medicine-related research [49-57]. However, our understanding of human diseases remains limited, the move from bedside to bench must continue to be pursued.

1 Zerhouni E. Medicine. The NIH Roadmap. Science, 2003, 302: 6372

2 Marincola F M. Translational medicine: A two-way road. J Transl Med, 2003, 1: 1

3 Geraghty J. Adenomatous polyposis coli and translational medicine. Lancet, 1996, 348: 422

4 Liu S H, Chu H I. Treatment of renal osteodystrophy with dihydrotachysterol (A.T.10) and iron. Science, 1942, 95: 388-389

5 Gong R, Ding C, Hu J, et al. Science, 2011, 333: 1642-1646

6 Zhang X W, Yan X J, Zhou Z R, et al. Arsenic trioxide controls the fate of the PML-RARalpha oncoprotein by directly binding PML. Science, 2010, 328: 240-243

7 Teng L, Zhao J, Wang F, et al. A GPCR/secretase complex regulates beta- and gamma-secretase specificity for Abeta production and contributes to AD pathogenesis. Cell Res, 2010, 20: 138-153

8 Pan X, Zhou T, Tai Y H, et al. Elevated expression of CUEDC2 protein confers endocrine resistance in breast cancer. Nat Med, 2011, 17: 708-714

9 Wang C, Huang Z, Du Y, et al. ATF4 regulates lipid metabolism and thermogenesis. Cell Res, 2010, 20: 174-184

10 Zhang J, Wang H, Xiao Q, et al. Hyaluronic acid fragments evoke Kupffer cells via TLR4 signaling pathway. Sci China Ser C-Life Sci, 2009, 52: 147-154

11 Zhao L, Galligan D C, Lamers S L, et al. High level HIV-1 DNA concentrations in brain tissues differentiate patients with post-HAART AIDS dementia complex or cardiovascular disease from those with AIDS. Sci China Ser C-Life Sci, 2009, 52: 651-656

12 Shan $\mathrm{C}, \mathrm{Xu} F$, Zhang S, et al. Hepatitis B virus X protein promotes liver cell proliferation via a positive cascade loop involving arachidonic acid metabolism and p-ERK1/2. Cell Res, 2010, 20: 563-575

13 Liu X, Leung S, Wang C, et al. Crucial role of interleukin-7 in T helper type 17 survival and expansion in autoimmune disease. Nat Med, 2010, 16: 191-197

14 Ding L, Xu Y, Zhang W, et al. MiR-375 frequently downregulated in gastric cancer inhibits cell proliferation by targeting JAK2. Cell Res, 2010, 20: 784-793

15 Cai Y, Shen X, Ding C, et al. Pivotal role of dermal IL-17-producing $\gamma \delta \mathrm{T}$ cells in skin inflammation. Immunity, 2011, 35: 596-610

16 Yang H, Wang J, Du J, et al. Structural basis of immunosuppression by the therapeutic antibody daclizumab. Cell Res, 2010, 20: 1361-1371
17 Chen S, Zhao J, Huang Y, et al. Endogenous enkephalin does not contribute to the cerebral anti-hyperalgesic action of gabapentin. Sci China Life Sci, 2010, 53: 1428-1432

18 Liu M, Wu H, Liu T, et al. Regulation of the cell cycle gene, BTG2, by miR-21 in human laryngeal carcinoma. Cell Res, 2009, 19: 828837

19 Qin J, Li R, Raes J, et al. A human gut microbial gene catalogue established by metagenomic sequencing. Nature, 2010, 464: 59-65

20 Bai X, Li P, Cao Y, et al. Engineering infectious foot-and-mouth disease virus in vivo from a full-length genomic cDNA clone of the A/AKT/58 strain. Sci China Ser C-Life Sci, 2009, 52: 155-162

21 Cao X J, Dai J, Xu H, et al. High-coverage proteome analysis reveals the first insight of protein modification systems in the pathogenic spirochete Leptospira interrogans. Cell Res, 2010, 20: 197-210

22 Wang J, Bai L, Li J, et al. Proteomic analysis of mitochondria reveals a metabolic switch from fatty acid oxidation to glycolysis in the failing heart. Sci China Ser C-Life Sci, 2009, 52: 1003-1010

23 Guo J, Sun Z, Xiao S, et al. Proteomic analysis of the cerebrospinal fluid of Parkinson's disease patients. Cell Res, 2009, 19: 1401-1403

$24 \mathrm{Li} \mathrm{Y,} \mathrm{Li} \mathrm{Z,} \mathrm{He} \mathrm{Y,} \mathrm{et} \mathrm{al.} \mathrm{Phylogeographic} \mathrm{analysis} \mathrm{of} \mathrm{human}$ papillomavirus 58. Sci China Ser C-Life Sci, 2009, 52: 1164-1172

25 Li S, Du H, Wang Z, et al. Meta-analysis of the relationship between Epstein-Barr virus infection and clinicopathological features of patients with gastric carcinoma. Sci China Life Sci, 2010, 53: 524-530

26 Zhang $\mathrm{C}$, Ding $\mathrm{N}$, Chen $\mathrm{K}$, et al. Complex positive selection pressures drive the evolution of HIV-1 with different co-receptor tropisms. Sci China Life Sci, 2010, 53: 1204-1214

27 Cao W, Yang Y, Wang Z, et al. Leukemia inhibitory factor inhibits T helper 17 cell differentiation and confers treatment effects of neural progenitor cell therapy in autoimmune disease. Immunity, 2011, 35: 273-284

28 Xiao T, Fan J K, Huang H L, et al. VEGI-armed oncolytic adenovirus inhibits tumor neovascularization and directly induces mitochondriamediated cancer cellapoptosis. Cell Res, 2010, 20: 367-378

29 Ying M, Zhen Q, Liu S, et al. Treatment of established colon carcinoma-bearing mice by dendritic cells pulsed with lysates of heat-treated tumor cells. Sci China Ser C-Life Sci, 2009, 52: 831-835

30 Dong L H, Wen J K, Miao S B, et al. Baicalin inhibits PDGF-BBstimulated vascular smooth muscle cell proliferation through suppressing PDGFR $\beta$-ERK signaling and increase in p27 accumulation and prevents injury-induced neointimal hyperplasia. Cell Res, 2010, 20: $1252-1262$

31 Wang G, Lu J. Retrovirus-mediated delivery of an IL-4 receptor antagonist inhibits allergic responses in a murine model of asthma. Sci China Life Sci, 2010, 53: 1215-1220

32 Zhao Y, Yang J, Shi J, et al. The NLRC4 inflammasome receptors for bacterial flagellin and type III secretion apparatus. Nature, 2011, 477: 596-600

33 Chen R, Zhang L, Zhong B, et al. The ubiquitin-specific protease 17 is involved in virus-triggered type I IFN signaling. Cell Res, 2010, 20: 802-811

34 Gu X, Yang X, Wang D, et al. Comparison and significance of specific and non-specific cellular immunity in patients with chronic hepatitis B caused by infection with genotypes B or C of hepatitis B virus. Sci China Ser C-Life Sci, 2009, 52: 719-723

35 Zhang H, Qiu Z, Jiao Y, et al. HIV-specific $\mathrm{CD}^{+} \mathrm{T}$ cell responses to HXB2 Gag and Nef peptide pools in Chinese HIV/AIDS patients. Sci China Ser C-Life Sci, 2009, 52: 841-846

36 Song X, Zhu S, Shi P, et al. IL-17RE is the functional receptor for IL-17C and mediates mucosal immunity to infection with intestinal pathogens. Nat Immunol, 2011, 12: 1151-1158

37 Chen H, Sun H, You F, et al. Activation of STAT6 by STING is critical for antiviral innate immunity. Cell, 2011, 147: 436-446

38 Liu X Y, Wei B, Shi H X, et al. Tom70 mediates activation of interferon regulatory factor 3 on mitochondria. Cell Res, 2010, 20: 9941011

$39 \mathrm{Hu}$ Y, Cong Y, Li S, et al. Identification of in vivo induced protein antigens of Salmonella enterica serovar Typhi during human infection. Sci China Ser C-Life Sci, 2009, 52: 942-948 
40 Sun P, Zhang Q, Han J, et al. TLR4 signaling induced TLR2 expression in the process of mimic cerebral ischemia/reperfusion in vitro. Sci China Life Sci, 2010, 53: 223-228

41 Liu S, Wang S, Wu Y, et al. Production of proinflammatory cytokines in the human THP-1 monocyte cell line following induction by Tp0751, a recombinant protein of Treponema pallidum. Sci China Life Sci, 2010, 53: 229-233

42 Ma F, Xu S, Liu X, et al. The microRNA miR-29 controls innate and adaptive immune responses to intracellular bacterial infection by targeting interferon- $\gamma$. Nat Immunol, 2011, 12: 861

43 Sun Y, Shi Y, Zhang W, et al. In silico characterization of the functional and structural modules of the hemagglutinin protein from the swine-origin influenza virus A (H1N1)-2009. Sci China Life Sci, 2010, 53: 633-642

44 Liu X, Zhan Z, Li D, et al. Intracellular MHC class II molecules promote TLR-triggered innate immune responses by maintaining activation of the kinase Btk. Nat Immunol, 2011, 12: 416-424

45 Mi S, Li Y, Yan J, et al. $\mathrm{Na}^{+} / \mathrm{K}^{+}$-ATPase b1 subunit interacts with M2 proteins of influenza $\mathrm{A}$ and $\mathrm{B}$ viruses and affects the virus replication. Sci China Life Sci, 2010, 53: 1098-1105

46 Li Z, Zhang Y, Liu Z, et al. ECM1 controls $\mathrm{T}(\mathrm{H}) 2$ cell egress from lymph nodes through re-expression of S1P(1). Nat Immunol, 2011, 12: $178-185$

47 Lei C Q, Zhong B, Zhang Y, et al. Glycogen synthase kinase 3b regulates IRF3 transcription factor-mediated antiviral response via activation of the kinase TBK1. Immunity, 2010, 33: 878-889

48 Shou Z, Xu Y, Xiao H, et al. The effect of pregnancy on paternal skin allograft survival. Sci China Ser C-Life Sci, 2009, 52: 1011-1015
49 Zhang D, Jiang W, Liu M, et al. Highly efficient differentiation of human ES cells and iPS cells into mature pancreatic insulin-producing cells. Cell Res, 2009, 19: 429-438

50 Ning J, Liu Q F, Luo X D, et al. Effect and mechanism of acute graft versus host disease on early diffuse murine lung injury following allogeneic stem cell transplantation. Sci China Ser C-Life Sci, 2009, 52: 1016-1022

51 Huang P, He Z, Ji S, et al. Induction of functional hepatocyte-like cells from mouse fibroblasts by defined factors. Nature, 2011, 475: 386-389

52 Gu T P, Guo F, Yang H, et al. The role of Tet3 DNA dioxygenase in epigenetic reprogramming by oocytes. Nature, 2011, 477: 606-610

53 Song Z, Cai J, Liu Y, et al. Efficient generation of hepatocyte-like cells from human induced pluripotent stem cells. Cell Res, 2009, 19: 1233-1242

54 Ren Z, Wang J, Zou C, et al. Comparative characterization of mesenchymal stem cells from different age groups of cynomolgus monkeys. Sci China Life Sci, 2010, 53: 563-572

55 Wang T, Chen K, Zeng X, et al. The histone demethylases jhdm1a/1b enhance somatic cell reprogramming in a vitamin-C-dependent manner. Cell Stem Cell, 2011, 9: 575-587

56 Li X, Zhu L, Yang A, et al. Calcineurin-NFAT signaling critically regulates early lineage specification in mouse embryonic stem cells and embryos. Cell Stem Cell, 2011, 8: 46-58

57 Lin J, Shi L, Zhang M, et al. Defects in trophoblast cell lineage account for the impaired in vivo development of cloned embryos generated by somatic nuclear transfer. Cell Stem Cell, 2011, 8: 371375

Open Access This article is distributed under the terms of the Creative Commons Attribution License which permits any use, distribution, and reproduction in any medium, provided the original author(s) and source are credited. 